the relative production of thorium to uranium because these elements are separated by only two atomic numbers. And the different decay rates of ${ }^{232} \mathrm{Th}$ and ${ }^{238} \mathrm{U}$ ensure that the abundance ratio of these two elements will be a sensitive function of their age. Cayrel $e t$ al. ${ }^{1}$ propose that the neutron-capture material in the atmosphere of CS31082-001 has an age of 12.5 Gyr with an uncertainty of 3.3 Gyr, a more accurate estimate of the age of the Universe. Further analysis of the whole range of neutron-capture elements in this star will refine this age estimate, narrowing the uncertainty.

We now know of a handful of stars born early in our Galaxy's history that are anomalously enriched in radioactive thorium, and at least one with uranium. We may expect to find more examples of such stars, as our surveys of the Galactic halo with the new generation of very large telescopes is just beginning. With new discoveries, more age estimates will be found, further nailing down the exact age of the Universe.

Christopher Sneden is in the Department of

Astronomy, University of Texas at Austin, Austin Texas 78712, USA.

e-mail: chris@verdi.as.utexas.edu

1. Cayrel, R. et al. Nature 409, 691-692 (2001).

2. Chaboyer, B. Phys. Rep. 307, 23-30 (1998).

3. Beers, T. C., Preston, G. W. \& Schectman, S. Astron. J. 103, 1987-2034 (1992).

4. Butcher, H. R. Nature 328, 127-131 (1987)

5. Sneden, C. et al. Astrophys. J. 533, L139-L142 (2000).

6. Westin, J., Sneden, C., Gustafsson, B. \& Cowan, J. J. Astrophys. J. 530, 783-799 (2000)

7. Cowan, J. J. et al. Astrophys. J. 521, 194-205 (1999).

8. Goriely, S. \& Clerbaux, B. Astron. Astrophys. 346, 798-804 (1999).

Evolution

\title{
Infectious speciation
}

Michael J. Wade

\section{The bacterium Wolbachia has strange and wonderful effects on} reproduction in its many invertebrate host species. In effect, the creation of new species can now be added to the list.

r or a new species to arise, a single population must somehow be split into two reproductively isolated populations that cannot interbreed. Such reproductive isolation usually stems from genetic incompatibility. It is easy to see how that arises when a geographical barrier divides one population of an organism into two, which then diverge genetically. On page 707 of this issue, however, Bordenstein, O'Hara and Werren $^{1}$ show that in two species of parasitoid wasp it is microbial infection that is the barrier to gene exchange.

The microbe concerned, Wolbachia pipientis, is a member of a highly diverse group of bacteria that is thought to include the ancestor of the mitochondrion - the powerhouse of multicellular organisms that was originally free-living. Wolbachia are endosymbionts, living inside the cells of certain host organisms, and like mitochondria they are almost always inherited through the maternal line. Their host range is broad, for the bacteria are found in association with about $20-75 \%$ of the insects, crustaceans, mites and nematode worms that have been surveyed with molecular markers ${ }^{2,3}$. Such is the range of effects of the microbe on its host - positive and negative - that it is not always possible to characterize Wolbachia simply as a mutualist, symbiont or pathogen.

Among the variety of reproductive anomalies caused by Wolbachia is the phenomenon of cytoplasmic incompatibility (Fig. 1), which results in the failure of infected host males and uninfected host females to produce offspring. Wolbachia

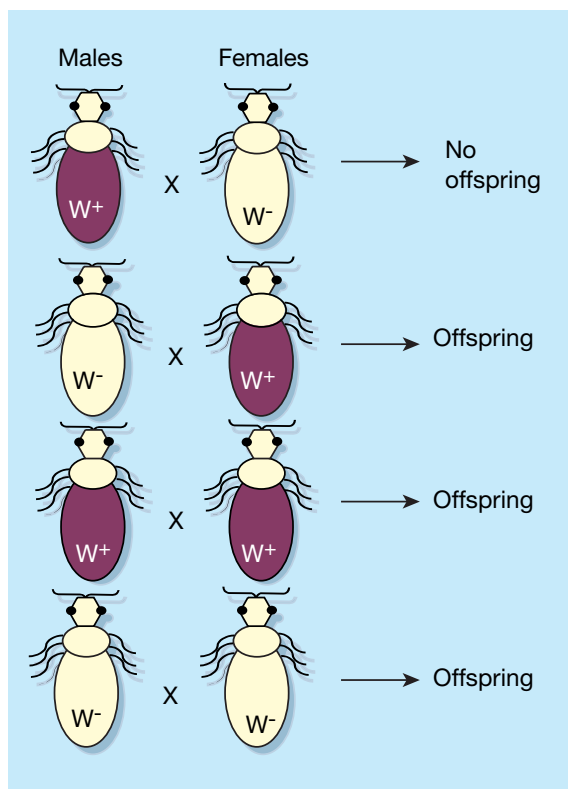

Figure 1 Wolbachia and cytoplasmic incompatibility. Cytoplasmic incompatibility means that when a male host infected with Wolbachia $\left(\mathrm{W}^{+}\right)$mates with an uninfected female $\left(\mathrm{W}^{-}\right)$, no offspring are produced. All other matings are fully compatible and result in the production of offspring. The consequence of this system is that the maternally transmitted Wolbachia tend to spread through the host species.

residing in host males are not typically transmitted to offspring, but they eliminate competing uninfected maternal lineages from the host population by their incompat-

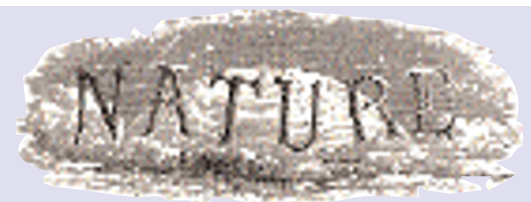

100 YEARS AGO

Dr. R. A. Daly, of the Department of Geology and Geography of Harvard University, is endeavouring to organise a geological and geographical excursion in the North Atlantic for the summer of 1901. Conditionally on the formation of a sufficiently large party, a steamer of about 1000 tons, specially adapted for ice navigation, and capable of accommodating sixty persons, will leave Boston on or about June 26... The main object of the voyage will be to offer to the members of the excursion party opportunity of studying the volcanic cones and lava-fields, the geysers, ice-caves and glaciers of Iceland, the fiords and glaciers of the west coast of Greenland, and the mountains and fiords of Northern Labrador... A hunting party may take part in the expedition; it could be landed for a fortnight or three weeks in Greenland and for about the same period in Labrador. From Nature 7 February 1901.

\section{YEARS AGO}

Surprisingly little of the information obtained with microscopes has been quantitative; most observers are content to sit at the microscope and regard the image, or to photograph it. Theoretically, it is possible to scan the image or its photograph mechanically; but this has seldom been done in practice. The whole method of obtaining resolution by lenses involves so much loss of light, lack of control of contrast, and other difficulties, that it is difficult to provide a good display or method of scanning. Some of these difficulties can be avoided by using a wholly different means of obtaining resolution and amplification. The essence of the problem of resolution is to separate in some way the light passing through very close regions of an object. The conventional microscope does this by using refraction by lenses to separate the light from neighbouring regions. An alternative method is to use the lens system the other way round, namely, to produce a minute spot of light. Discrimination between neighbouring points is then produced by passing the light through them at different times by making the spot scan it. After passing through the preparation, the spot is made to fall on a photocell, with subsequent amplifcation and display as required. Such a flying-spot microscope depends on scanning different parts at different times, and will only give accurate information about objects that are stationary or moving only at a rate of a different order from that of the spot. From Nature 10 February 1951. 
ible matings. So the bacteria in males are essential to the spread of their maternally transmitted relatives through the host population.

Typical cytoplasmic incompatibility falls short of speciation because the barrier to reproduction between infected and uninfected populations works only in one direction, not reciprocally. Although females of the uninfected host population cannot interbreed with males of the infected host population, the reciprocal cross is fully fertile. But there is evidence ${ }^{4,5}$ that different genetic strains of Wolbachia can cause reciprocal, two-way reproductive isolation between host populations in some parasitoid wasps, mosquitoes and fruit flies ${ }^{6}$. This observation has led some evolutionary biologists to speculate that Wolbachia might be an agent of infectious speciation ${ }^{6,7}$.

Such speculation is controversial, for two reasons. First, it is widely accepted that, when two host populations become reproductively isolated, so do the populations of their respective endosymbionts. Hence, in a process called co-speciation, a host may cause subsequent speciation of its endosymbionts, an explanation suggested for the genetic divergence of strains of Wolbachia ${ }^{8}$. The hypothesis of infectious speciation turns this view on its head. Second, so the theory goes, speciation occurs when reproductive isolation arises as the incidental by-product of the gradualistic, genetic divergence of two populations. Microbial speciation, in contrast, might be comparatively rapid (as seen for instance in polyploid or hybrid speciation in some plants ${ }^{9}$ ), and could occur without any genetic evolution of the host. Polyploid speciation occurs through a doubling, or more, of chromosome number.

Bordenstein and colleagues ${ }^{1}$ provide evidence that microbes have acted faster than genes in producing reproductive isolation between the wasps Nasonia giraulti and $N$. longicornis; this can be taken as the first stage of speciation. First, the authors showed that each wasp harbours a genetically distinct strain of Wolbachia that causes cytoplasmic incompatibility with the other uninfected host species. They then used antibiotics to create an uninfected strain of each host species and demonstrated that in Wolbachiafree wasps there are no genetic barriers in first- or second-generation hybrids to free interbreeding between the two wasps.

How might these findings fit into a standard genetic model of speciation, as shown in Fig. $2 a$ ? In this model, incompatible gene combinations (such as $A_{1} B_{1}$ ) cause sterility or inviability of offspring, and so speciation. Events begin with an ancestral species, $\mathrm{A}_{0} \mathrm{~A}_{0} \mathrm{~B}_{0} \mathrm{~B}_{0}$, that becomes split by geological events into two geographically isolated daughter populations. The evolutionary forces of mutation, random genetic drift

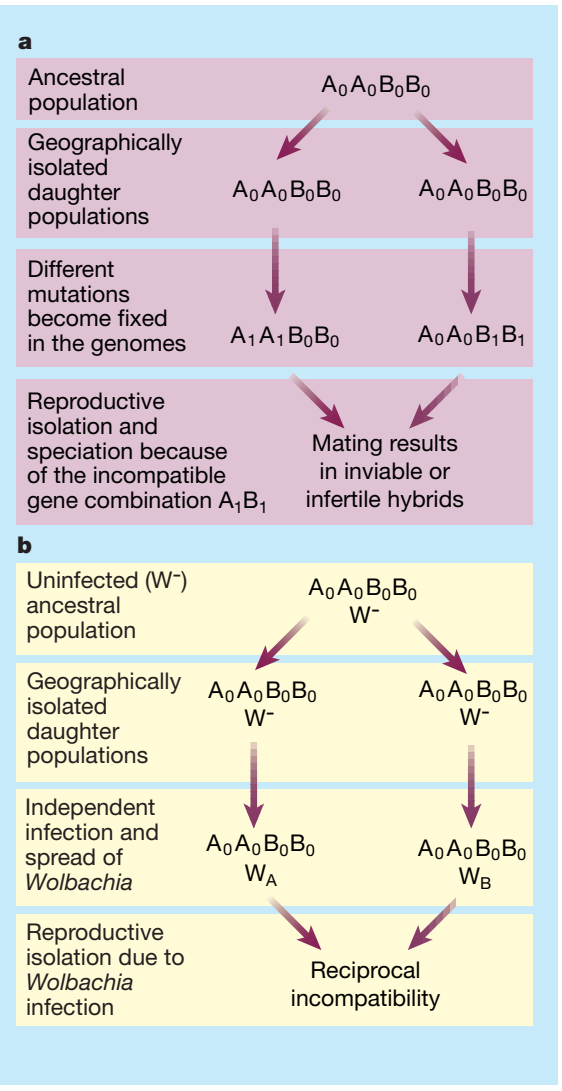

and natural selection operate independently on each daughter population. Eventually, one gene undergoes mutation to allele $A_{1}$, and becomes fixed in one daughter population, while a second mutation, to allele $\mathrm{B}_{1}$ at the other gene, becomes fixed in the second daughter population. The two daughter populations become reproductively isolated because matings between them result in the $A_{1} B_{1}$ deleterious gene combination. In this classic model, genetic barriers to reproduction and genetic exchange, and so speciation, arise as a by-product of local, gradual evolution.

Microbially driven speciation could occur in much the same way, stemming from cytoplasmic incompatibility between two different strains of Wolbachia infecting the same host species (Fig. 2b). Here, however, infectious transmission of incompatible Wolbachia strains, one in each daughter population, replaces the incompatible gene combinations. Predatory mites and parasitoid wasps are the most likely candidates for spreading Wolbachia between different species of host. Previous cases of reciprocal cytoplasmic incompatibility have been between species pairs, which also exhibited evidence of genetic barriers to gene exchange. Whenever both are present, it is difficult to determine which - the incompatible gene combinations or the microbes - came first. The report by Bordenstein et al. provides evidence that, at least in this case, microbially induced reproductive isolation preceded genetic isolation.
Figure 2 Genetic and infectious models of speciation. a, A standard genetic model in which the initial state is an ancestral population of a species that is homozygous at both of two gene loci, and so is $A_{0} A_{0} B_{0} B_{0}$. Following geographical isolation, each of two daughter populations is gradually modified as new alleles $\left(A_{1}\right.$ and $\left.B_{1}\right)$ arise by mutation and then become fixed in the genome by random genetic drift and natural selection. Because the $A_{1} B_{1}$ gene combination causes complete inviability or sterility in hybrids, the daughter populations are new, descendant species. $b$, Infectious speciation, which parallels the genetic model. The initial state is an ancestral species, $\mathrm{W}^{-}$, not infected with Wolbachia. Two daughter populations arise which have become infected by different strains of Wolbachia (A and B) after transmission from a parasite or parasitoid. The different strains then become fixed in each genome by cytoplasmic incompatibility. Reciprocal cytoplasmic incompatibility between $\mathrm{W}_{\mathrm{A}}$ males and $\mathrm{W}_{\mathrm{B}}$ females, and $\mathrm{W}_{\mathrm{B}}$ males and $\mathrm{W}_{\mathrm{A}}$ females, prevents hybridization, so in effect the daughter populations are new species even though they remain genetically identical to one another and to the ancestor.

How common might infectious speciation be? It is not possible to draw a conclusion from this single example - which has of course to be contrasted with the many examples of genetic speciation ${ }^{10}$. But there are several reasons why it is unlikely to happen often. First, incomplete cytoplasmic incompatibility (where incompatible crosses produce some progeny instead of none) seems to be more common than complete cytoplasmic incompatibility. Reciprocal but incomplete incompatibility is not a barrier to gene flow. Second, genetic models of Wolbachia-host coevolution indicate that the favoured trajectory is from complete to incomplete cytoplasmic incompatibility. Finally, we know little of the initial stages of Wolbachia infection in natural populations. When artificially introduced into new hosts, Wolbachia can be difficult to transmit ${ }^{11}$. So the experimental results are consistent with the scheme outlined in Fig. 2b, but may not reflect the actual historical sequence of events.

Nevertheless, with the paper by Bordenstein et al., host speciation can now be added to the list of modifications to reproduction caused by Wolbachia infection. Given the ubiquity of Wolbachia, infectious barriers to gene exchange may be much more common in the early stages of speciation than we realize.

Michael J. Wade is in the Department of Biology,

Indiana University, Bloomington, Indiana 47405 , USA.

e-mail:mjwade@bio.indiana.edu 
1. Bordenstein, S. R., O’Hara, F. P. \& Werren, J. H. Nature 409, 707-710 (2001).

2. Werren, J. H., Windsor, D. \& Gao, L. Proc. R. Soc. Lond. B 262, 197-204 (1995).

3. Jeyaprakash, A. \& Hoy, M. A. Insect Mol. Biol. 9, 393-405 (2000).

4. Wade, M. J., Chang, N. W. \& McNaughton, M. Heredity 75, 453-459 (1995).

Shoemaker, D. D., Katju, V. \& Jaenike, J. Evolution 53, 1157-1164 (1999).
6. Hurst, G. D. D. \& Schilthuizen, M. Heredity 80, 2-8 (1998).

7. Thompson, J. N. Biol. J. Linn. Soc. 32, 385-393 (1987).

8. Futuyma, D. J. Evolutionary Biology 541 (Sinauer, Sunderland, MA, 1998).

9. Reiseberg, L. H. Annu. Rev. Ecol. Syst. 28, 359-389 (1997).

10. Wu, C. I. \& Palopoli, M. F. Annu. Rev. Genet. 28, 283-308 (1994).

11.Clancy D. J. \& Hoffmann, A. A. Am. Nat. 149, 975-988 (1997).

\section{Astronomy}

\section{The day the solar wind nearly died}

Mike Lockwood

On 11 May 1999, the density of the solar wind dropped almost to zero. Space scientists are now giving their first reports of this rare opportunity to study the complex relationship between the Sun and Earth.

$\mathrm{T}$ he study of space is generally passive, as the input factors to an environment cannot be adjusted in a controlled manner to study one isolated mechanism, as they can in a laboratory. Instead scientists have to monitor all the inputs and try to disentangle the various effects that are taking place simultaneously. For instance, the Sun emits a continuous stream of ionized gas (containing mostly protons and electrons) called the solar wind, which varies in concentration, flux, speed, temperature and composition. All of these factors affect the magnetosphere - the cavity formed by the Earth's magnetic field in the solar wind - and separating their various effects is difficult. This is why rare events such as the one centred around 11 May 1999 are so valuable. In this period, the solar wind remained completely normal except that its density plummeted to $5 \%$ of

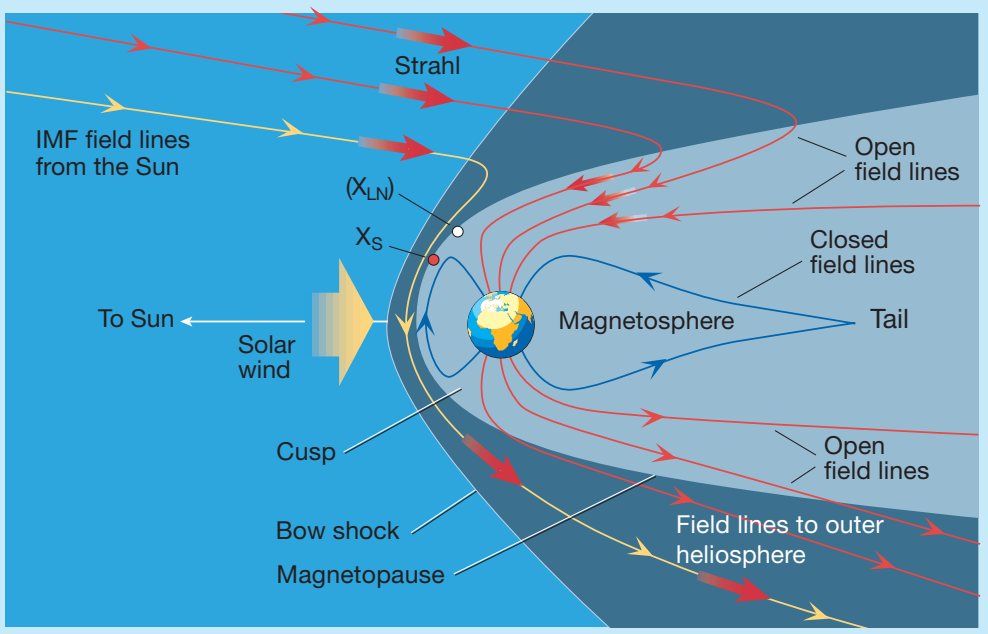

b

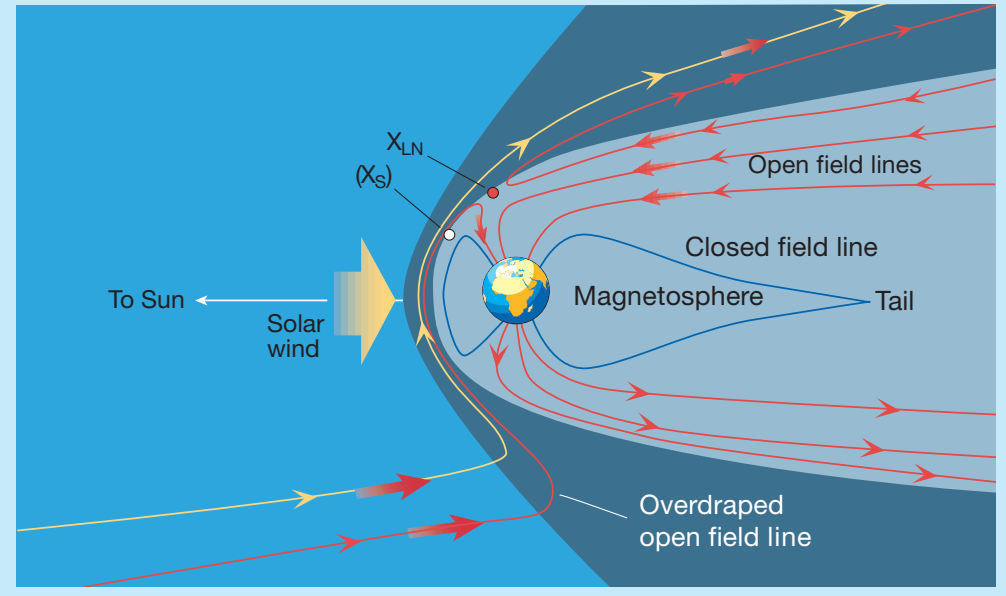

typical values. The first studies from this period are now published in a special issue of Geophysical Research Letters'.

When the density dropped, many aspects of the magnetosphere's behaviour were as scientists had predicted, which was a satisfying triumph for current theories. But the event also had some puzzling characteristics. Some of these are apparent in the data presented in these initial papers, although not all are commented on. Others aspects are so intriguing that further study is required.

Earth's magnetic field is confined to the low-density, high-field magnetosphere by the dynamic pressure of the solar wind on the side of the Earth facing the Sun, and by thermal pressure on the long tail that trails away from the Sun (Fig. 1). Both these pressures depend on the concentration of the solar wind, so the magnetosphere grew to exceptionally large dimensions (100 times its typical volume) as the solar wind decayed. Another feature was the appearance of highly energetic flows of electrons parallel to the direction of the magnetic field in the vicinity of Earth. These so-called 'strahl' electrons (red arrows in Fig. 1) are continuously emitted by the Sun but their flow is usually disrupted by the solar wind, making their fluxes

Figure 1 Earth's magnetosphere and the solar wind. $a$ and $b$ show two possible ways in which the interplanetary magnetic field (IMF) can interconnect with Earth's magnetospheric field. a, New open field lines (red lines) are produced at a reconnection site $X_{s}$ and solar wind energy is directly deposited in the inner magnetosphere and upper atmosphere, as well as being stored in the tail of the magnetosphere because open field lines accumulate there. b, Field lines that are already open are reconfigured by reconnection at $\mathrm{X}_{\mathrm{LN}}$, in this example in the Northern Hemisphere. In this instance, solar-wind energy is not added to the tail because no new open flux is produced. Closed field lines are shown in blue; unconnected IMF lines are yellow; strahl electrons are represented by red arrows. The magnetopause is the boundary between the magnetosphere and the solar wind, and the bow shock is the edge where the supersonic solar wind abruptly drops in velocity. The solar wind behind the bow shock (dark blue) is denser than the incoming solar wind (medium blue), whereas the magnetosphere (grey) is the least dense of the three regions. A study of Earth's magnetosphere during a period of exceptionally low solar-wind flux promises to explain the complex interplay between these two situations ${ }^{1}$. 